\title{
Students work like astroparticle physicists with Cosmic@Web
}

\author{
Philipp Lindenau, ${ }^{a, *}$ Carolin Schwerdt ${ }^{b}$ and Michael Walter ${ }^{b}$ \\ ${ }^{a}$ Technische Universität Dresden \\ Institute of Nuclear and Particle Physiks \& Chair of Didactics of Physics \\ Germany \\ ${ }^{b}$ DESY Zeuthen \\ Germany \\ E-mail: philipp.lindenau@tu-dresden.de, carolin.schwerdt@desy.de, \\ michael.walter@desy.de
}

Cosmic@Web is an online learning resource developed at DESY in Zeuthen, Germany as part of the outreach activities in the framework of Netzwerk Teilchenwelt. Via Cosmic@Web, high school and university students can access data from astroparticle physics experiments and experience the workflow of scientific research in this field by pursuing their own or suggested research questions. Data from various experiments located in different areas of the world can be used to study cosmic weather effects and muon properties. The analysis can be performed without any coding experience. The graphical interface allows to visualize data in several plot types and offers possibilities of data fitting as well as data reduction and correction. So far, Cosmic@Web has been used by German high school students during internships at research institutes like DESY, for a research component as part of their high school degree as well as within projects in software development and coding. Additionally, connections to established contents of high school physics curricula will be pointed out and the design of a Cosmic@ Web workshop for high school students and teachers will be presented. Furthermore, the acceptance of the tool by students and teachers as well as their feedback during and after its introduction in the workshops will be discussed.

Keywords: astroparticle physics, data analysis, education, school, workshop

$37^{\text {th }}$ International Cosmic Ray Conference (ICRC 2021)

July 12th - 23rd, 2021

Online - Berlin, Germany

\footnotetext{
${ }^{*}$ Presenter
} 


\section{Netzwerk Teilchenwelt brings particle physics to school}

Since its founding in 2010, Netzwerk Teilchenwelt has continuously increased its outreach activities with the goal to make aspects of fundamental research in particle, astroparticle and lately also hadron and nuclear physics accessible to high school students, teachers and the general public [1]. Key activities in pursuing this mission are Masterclasses as well as workshops and research programs for high school students at research institutes like CERN, the development of teaching material and textbooks ${ }^{1}$ and the offering of in-service teacher trainings.

The astroparticle physics activities of Netzwerk Teilchenwelt are coordinated and supervised at DESY in Zeuthen. One goal is the development of experiments for the measurement of cosmic radiation which are designed to be used by high school students, such as the CosMO detector [2] and of complementary teaching material [3]. In addition, DESY has realized the online learning toolCosmic@Web. It enables the online analysis of real data from various experiments with cosmic particles (see section 2).

So far, high school students have used Cosmic@Web during internships at research institutes, to compare own measurements taken with the CosMO experiment with Cosmic@Web data, for special learning activities as a part of their high school degree, for the preparation of a technical paper, for own projects on programming and software development ${ }^{2}$, while participating in the International Cosmic Day [4] and in workshops (see section 4).

\section{Essential contents of Cosmic@Web}

Essential aspects of the structure and design of Cosmic@Web will be outlined here. Further information can be found in [5].

The data provided within Cosmic@Web can be accessed via a web browser on a computer, a tablet or even a smartphone. To enable students to work and learn independently, two websites have been created. The website https://cosmic.desy.de/eng gives an introduction to the subject and provides the necessary information to understand the experiments and data sets as well as a description of the different options for data analysis. The analysis tool can be found on the second website https://cosmicatweb.desy.de.

All data sets in Cosmic@Web consist of experimental data from long-term measurements, mostly continuously over eight years. The detectors are partly installed at DESY in Zeuthen but also at other locations such as the German research vessel Polarstern which regularly travels between the Arctic and the Antarctic Oceans, from 2013 to 2017 on the German research station Neumayer $I^{3} I^{3}$ in Antarctica and since 2019 on the Zugspitze (Germany, $2656 \mathrm{~m}$ ). Two detectors within the SEVAN network ${ }^{4}$ (Mt. Aragats in Armenia, $3200 \mathrm{~m}$ and Mt. Musala in Bulgaria, $2925 \mathrm{~m}$ ) have been detecting muons, electrons, positrons, gammas and neutrons since 2012. The data within Cosmic@Web includes, for example, over five million muon decays and 12 passings of the equator by the Polarstern.

\footnotetext{
${ }^{1}$ https://www.teilchenwelt.de/material/materialien-fuer-lehrkraefte

${ }^{2}$ For example the tutorial of Cosmic@Web was programmed by high school students.

${ }^{3}$ https://www.awi.de/en/expedition/stations/neumayer-station-iii.html

${ }^{4}$ http://crd.yerphi.am/SEVAN
} 
No data reduction has been performed. The reasonable data reduction and subsequent analysis as well as the interpretation of the data are essential work steps that are carried out by the learners themselves. Data sets for nine experiments are offered.

Since no elaborated coding skills can be expected from high school students, a web-based graphical user interface has been created that provides various tools for data analysis. A tutorial is offered in which a diagram is created under guidance. In addition, sample diagrams can be loaded for different experiments. Cosmic@Web offers a standard mode for beginners and an advanced mode for experienced users. One can choose between German and English websites. After the selection of the evaluation criteria and plot options, the data is displayed in a diagram and it can be saved as an image or online by assigning a session ID. E-mail exchange and video conferences with scientists at DESY are offered for support.

\section{Why to work with Cosmic@Web?}

Cosmic@Web allows students to work like astroparticle physicists. It was and is being developed in collaboration with teachers and high school students and their valuable feedback has been taken into account to a great extent. Below, selected possible contributions of Cosmic@Web to high school education will be addressed.

\section{Connection to physics curricula}

Speaking specifically for Germany, particle and astroparticle physics is not an explicit content of the high school curriculum in every state. Nevertheless, Cosmic@Web offers many points of contact with established contents of physics curricula. Examples are the relativistic time dilation which can be investigated by analyzing the lifetime of cosmic muons. By analyzing data of the muon detector and the mini-neutron-monitor at the Polarstern in dependence of the latitude position (see section 4), the behavior of electrically charged particles in the Earth's magnetic field, the particle flux from the Sun and, with additional data from the SEVAN network, cosmic weather effects can be studied. Furthermore, the technology of the detectors offers examples of the application of semiconductor technology, scintillation, optical fibers and the photoelectric effect.

\section{Fostering essential competencies}

The use of Cosmic@Web as a digital learning tool offers many opportunities to meet the requirements of modern and competence-oriented physics teaching. Learning with digital media and the internet connects the reality of teenagers' lives with physics tasks [6]. Competencies in the area of knowledge acquisition can be decisively promoted by the wide range of open learning opportunities and the possibility of independent scientific work with authentic data offered by Cosmic@Web. Communication skills are further developed during the presentation of results in talks and posters as well as in scientific papers. Examples of these results can also be found on the Cosmic@Web website.

\section{Motivational aspects}

Results of the ROSE study show that topics related to the universe and open research questions are likewise interesting to German boys and girls [7]. This interest might be supported in the future by anticipated achievements in the emerging field of multi-messenger astronomy and the associated 
media attention. From this point of view astroparticle physics seems like a suitable and promising context to generate and foster students' interest in natural sciences and physics specifically.

Since for every experiment data from several years is available, many students can work on the same topic but with different data sets and compare the results. This provides room for discussion which is especially beneficial for girls' interest in physics [8]. Due to the diverse possibilities offered withinCosmic@Web regarding research questions, the deepening of technical knowledge or the expansion of the level of analysis, the heterogeneity in knowledge among students can be well addressed and individual, explorative learning tailored to the interests of the learners can be promoted.

\section{Authentic scientific work}

One goal of Cosmic@Web is to enable students to understand and reconstruct scientific work. Experimental work is a constant interplay between building hypotheses, analyzing data, interpreting diagrams, reading up on basics and further investigation based on the newly gained knowledge. In the end, the validity of the hypothesis that has been established is tested and results can be published. With the wide range of data sets and analysis tools within Cosmic@Web this workflow can be well experienced.

\section{Working with Cosmic@Web - hypothesis generation and testing in a workshop}

In this section, the concept of a digital workshop will be described which is supposed to enable students and teachers to get started with Cosmic@Web and give them an idea how they can conduct further research after the workshop. First implementations have shown that Cosmic@Web is also suitable for use with a larger audience rather than for individual research only. Here only the hands-on work with Cosmic@Web will be described in detail. During the workshops additional information on the key ideas of astroparticle physics, on general research questions and experiments was provided. For the students the description of the cycle of scientific research (see section 3) was another essential part of the workshop, whereas in the workshops with teachers the focus was on the use of Cosmic@Web and the discussion of astroparticle physics in class. The hands-on activities during the workshops were the same for teachers and students.

In the following, the process of hypothesis generation and testing will be outlined on the example of data from the research vessel Polarstern which usually travels between the Arctic and Antarctic Oceans and has recently attracted scientific and media attention for the MOSAiC expedition. ${ }^{5}$

The first part of the hands-on activities was the execution of the tutorial. There the learners were guided through the creation of a diagram that shows the measured muon rate as a function of time. The data set used includes Polarstern data from a whole year. The participants were paired in groups of three to four and had the task to complete the tutorial, discuss the result and to form hypotheses on how the visible data distribution (figure 1) could be explained. ${ }^{6}$ The hypotheses that were stated to explain the fluctuation of the muon rate were very diverse and ranged from the influence of solar activity, the Sun-to-Earth distance, the seasons of the year or even the influence of

\footnotetext{
${ }^{5}$ https://www.awi.de/en/focus/mosaic-expedition.html

${ }^{6}$ It is planned to update the tutorial so a newer data set is being used which is taken with better detectors leading to smaller systematic errors. Furthermore, the plot will be changed to display only the data points without linear connections.
} 
ocean waves or the day-night cycle up to the influencing factor to be further investigated during the workshop: the position of the Polarstern on its journey between the Arctic and Antarctic Oceans. The hypotheses were not judged or discussed individually and giving a precise physical explanation was not the goal either at this point but for the participants to actively comprehend the process of hypothesis generation in the scientific research process. The major questions of the participants concerning the plot were about the time dimension of the diagram ${ }^{7}$ (which led to the wave motion and day-night cycle hypotheses) and the parts of the diagram where there is no data visible. ${ }^{8}$

Starting from those explanation attempts the focus was put on the variable position of the ship, which is unique for this experiment, with the goal to investigate the dependence of the muon rate on this position. As a first step, a map plot was created that showed the ship's position as a function of time for the same data set as used before (figure 2, left). This plot type is rather easy to interpret and does not require much additional explanation as is the case for other available types such as histograms. Furthermore, the inclusion of a third variable was introduced, which is visualized via a color code. ${ }^{9}$ The participants again created the plot themselves

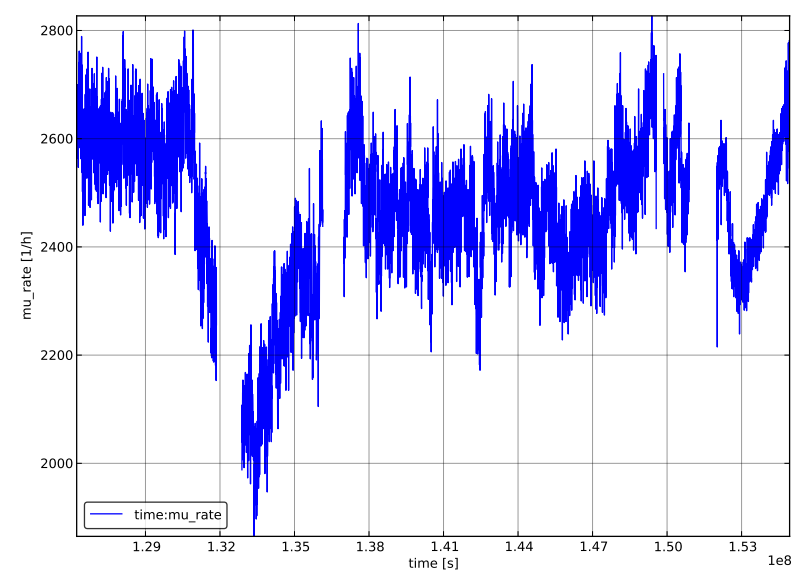

Figure 1: The result of the Cosmic@Web tutorial. A diagram of the muon rate as a function of time for one year of data acquisition. following the instructions of the lecturers.

The interpretation of the diagram was done in the plenum to make sure the reading of the color coding was clear. The route of the Polarstern was reconstructed from the plot and afterwards those insights were linked to the fluctuation of the muon rate that has been seen in the tutorial diagram (figure 1). After interpreting the diagram and reconstructing the route, the question was posed, how the analysis should be changed to actually investigate the dependence of the muon rate on the ship's position and together the diagram was adjusted accordingly by changing the third variable to be the muon rate. The result is shown in figure 2 on the right.

In this plot one can see that the muon rates by trend are higher in the pole regions and lower in the equator region. This observation then was connected to the students' prior knowledge on the magnetic field of the Earth and the behavior of electrically charged particles in magnetic fields, naming polar lights as another well-known phenomenon related to the matter. Furthermore, revising the earlier imposed questions on the missing data in the tutorial plot, the regions were identified where the detector was not taking data. The analysis of the plot again brought up new questions, such as: Why do the muon rates differ in the same region, as can be seen west of Africa and why is there a region near the tip of Africa where the muon rate is comparatively low? Going from there, it was discussed which other parameters could influence the muon rate by simultaneously getting

\footnotetext{
${ }^{7}$ The time is displayed in $10^{8}$ seconds counted since the 1st of January 2010, 00:00 UTC.

${ }^{8}$ Those are simply periods where the detector was not working.

9In this plot type the two other variables are the ship's position coordinates.
} 

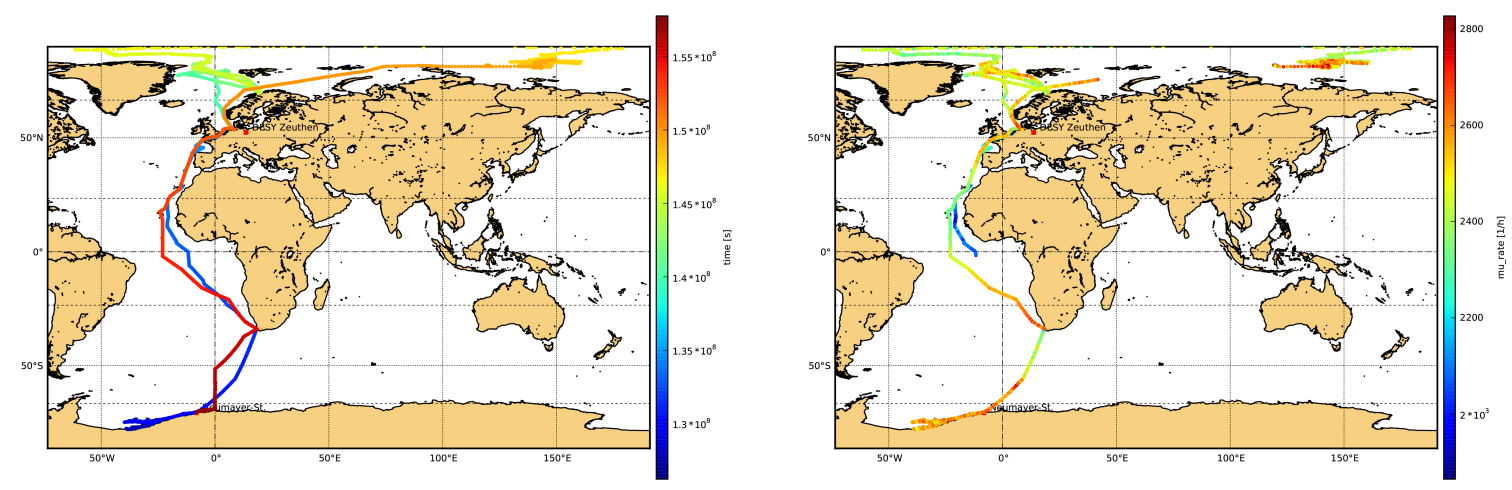

Figure 2: Diagrams of the ship's position in dependence of time (left) and the muon rate as a function of the ship's position (right).

back to earlier hypotheses such as a potential impact of the Sun's activity. Moreover, influences of air pressure and of the inhomogeneity of the Earth's magnetic field were briefly pointed out with the remark that many possible influences can be investigated with the data available in Cosmic@Web by changing variables and plot types, applying data reduction, comparing data sets from different years and taking into account data from other experiments. With this outlook on possible analysis methods the introduction to Cosmic@Web ended. The participants have produced and interpreted different diagrams and have generated hypotheses every time, for some of which first steps have been made to test them, leaving room for future individual research.

\section{Students' and teachers' feedback}

So far, the digital workshop has been carried out twice with school students (grades 9 to 13, duration of 120 minutes) and four times with teachers (duration between 90 and 120 minutes). After the events the participants were asked for their feedback in form of a questionnaire. Among other things, they were asked to state to which extent given statements apply to them. The given options were: 1. absolutely not true, 2. rather not true, 3. partly true and partly not, 4. rather true, 5. absolutely true.

Figure 3 shows the distribution of participants' responses for 8 selected items of the questionnaire. The associated statements read as follows:

(1) I enjoyed the data analysis with Cosmic@Web.

(2) The data analysis with Cosmic@Web was interesting for me.

(3) My idea of how a physicist works changed during the workshop.

(4) While working with Cosmic@Web, I felt like a real researcher. (only students)

(5) I would like to investigate further research questions independently with Cosmic@Web after the workshop.

(6) I considerCosmic@Web suitable for use in scholar education. (only teachers)

(7) I would like to use Cosmic@Web in my classes. (only teachers)

(8) For my teaching I see a possibility to use Cosmic@Web. (only teachers) 


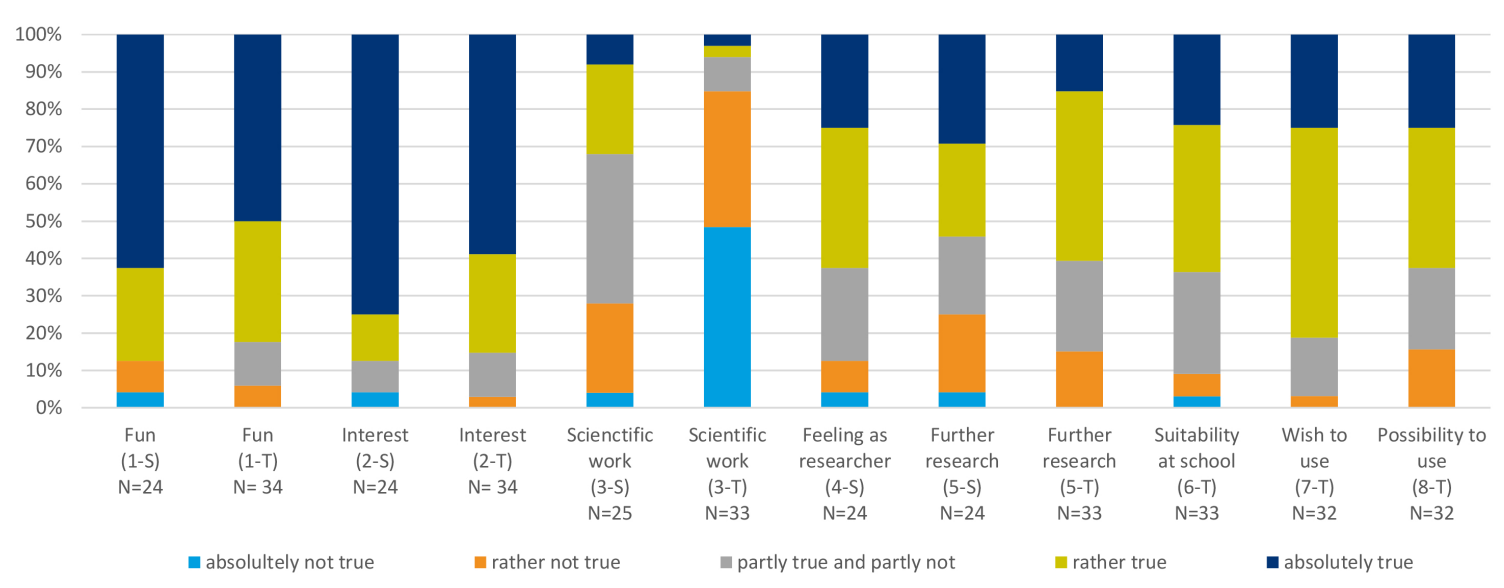

Figure 3: Distribution of participants' responses to selected items of the evaluation questionnaire labeled with the item number in brackets (S: students, T: teachers; N: total number of answers). For all items the option "absolutely true" (dark blue) represents the most positive response.

Although the sample sizes are rather small, the feedback is encouraging and additional open text items provided hints in which respects the workshop and Cosmic@ Web itself could be further improved. It should be mentioned that the participation in the workshop was voluntary and therefore it has to be assumed that the participants already were interested in astroparticle physics before the workshop. The data shows that most students and teachers enjoyed the hands-on activities with Cosmic@Web and found them interesting $(1,2)$. As expected, the teachers' view on the scientific workflow in physics hardly changed, but for the students data suggests that their view changed to some extent (3) and many had at least partly the feeling of pursuing scientific research when working with Cosmic@Web (4), which is one of its main goals. Many students as well as teachers stated their intention to investigate research questions independently with Cosmic@Web after the workshop (5).

In the pastCosmic@Web proved to be suitable for supporting research projects of individual students. The presented workflow outlined opportunities how Cosmic@Web can be used in class also with a larger group of learners, especially for triggering interest in conducting research (5). For this to happen, Cosmic@Web has to be actually used by teachers or at least recommended to their students. Therefore, teachers' perception of the usability of Cosmic@Web in class (6) as well as their motivation to really use it (7) and, of course, their perception of the possibility to do so within their respective scholar framework (8) were important aspects in the evaluation. The teachers' responses show that the majority sees possibilities to integrate Cosmic@Web into their teaching and also wants to do so because Cosmic@Web to their mind showed suitable for in-class use. The main concern of those teachers who do not see good chances to use Cosmic@Web is their respective curriculum. Considering the fact that Cosmic@Web was designed to be as authentic as possible, leading to compromises in tailoring it to scholar needs while not drifting away too far from the common research practice in the field, we consider the teachers' responses to items (6) to (8) as satisfactory.

The workshop in general seems to be appropriate to meet the needs of teachers and students. This perception is further supported by the fact that 30 out of 33 teachers and 19 out of 23 students 
stated that they would recommend the workshop to colleagues or friends respectively while the rest stated not to know if they would. The evaluation results can be regarded as a proof of concept for digitalCosmic@Web workshops for teachers and students with room for improvement, especially in the design for teachers.

\section{Planned adaptions}

The workshop duration for students appeared to be suitable to give a brief introduction to astroparticle physics and into the workflow and possibilities with Cosmic@Web. Future workshops for teachers should be extended by at least 45 minutes, as can also be deduced from the feedback. By doing so, the requests for more examples and a broader introduction to astroparticle physics can be taken into account and concerns from the teachers' side considering the complexity of Cosmic@Web may be countered by a more detailed overview of the contents of the support pages that provide necessary background information for teachers as well as students.

\section{Acknowledgments}

We would like to thank Gesamtmetall - Nachwuchssicherung/think $I N G^{10}$ for supporting the development of Cosmic@Web, the Dr. Hans Riegel-Stiftung ${ }^{11}$ for their continuous financial support of the teacher trainings of Netzwerk Teilchenwelt, the German Federal Ministry of Education and Research for funding activities of Netzwerk Teilchenwelt in the project KONTAKT and, of course, everyone who provided feedback during the development and testing of Cosmic@Web.

\section{References}

[1] Bilow, U., Kobel, M. (2020). Netzwerk Teilchenwelt: Coordinated Outreach and Recruitment of Young Talents in Germany. PoS(ICHEP2020)936

[2] Franke, R. et al. (2013). CosMO - A Cosmic Muon Observer Experiment for Students. arXiv:1309.3391 [astro-ph.IM]

[3] Karg, T. et al. (2016). Teilchenphysik. Unterrichtsmaterial ab Klasse 10. Kosmische Strahlung. Hamburg: Joachim Herz Stiftung

[4] Hütten, M. et al. (2017). The International Cosmic Day - An Outreach Event for Astroparticle Physics. PoS(ICRC2017)405

[5] Garrappa, S. et al. (2019). Cosmic@Web - Astroparticle learning platform for students. PoS(ICRC2019)419

[6] Deutsche Physikalische Gesellschaft (2016). Physik in der Schule: Hauptteil. Bad Honnef

[7] Elster, D. (2007). Student interests - the German and Austrian ROSE survey. Journal of Biological Education, 42(1), p. 5-11

[8] Hoffmann, L., Häußler, P. and Lehrke, M. (1998). Die IPN-Interessenstudie Physik. Kiel: IPN

\footnotetext{
${ }^{10}$ https://www.think-ing.de

${ }^{11} \mathrm{https} / / /$ www.hans-riegel-stiftung.com/
} 